RICYDE. Revista Internacional de Ciencias del Deporte doi: $10.5232 /$ ricyde

Rev. int. cienc. deporte

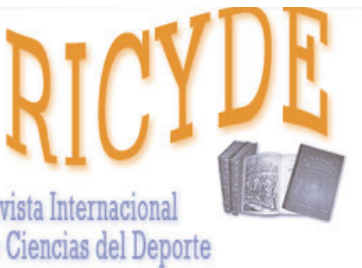

RICYDE. Revista Internacional de Ciencias del Deporte VOLUMEN XII - AÑO XII

Páginas:149-163 ISSN:1885-3137 Número 44 - Abril - 2016

\title{
Efecto de la localización del partido en eliminatorias de ida y vuelta de la UEFA Champions League
}

\section{Influence of match locating in UEFA Champions League two-legged ties}

\author{
Miguel Pic $^{1}$ y Julen Castellano ${ }^{2}$
}

1. Universidad de La Laguna; IES Garoé. España

2. Universidad del Pais Vasco (UPV/EHU). España

\section{Resumen}

El presente trabajo tiene como objetivo indagar sobre la ventaja de jugar en casa eliminatorias a doble partido en fútbol. Fueron analizadas eliminatorias de cuartos de final y semifinal de la UEFA Champions League desde 1954-55 hasta 2013-14, haciendo un total 642 partidos y 336 eliminatorias. Se aplicó un contraste no paramétrico binomial para conocer la influencia de jugar en casa, mientras que la regresión logística binaria se utilizó para mostrar la proporción explicativa de los goles al pasar la eliminatoria tras el primer enfrentamiento. Los principales resultados mostraron que en cuartos de final, los equipos locales (segundo partido) pasaron la eliminatoria en mayor proporción que en semifinales, en las que los equipos visitantes (segundo partido) se clasificaron en mayor proporción. En un plano estratégico, una mayor adecuación del modelo de juego y la selección de alineaciones más acordes con el orden de eliminatorias a doble partido serán algunas utilidades ofrecidas por este trabajo para los entrenadores.

Palabras clave: ventaja de jugar en casa; fútbol; competición; UEFA

\begin{abstract}
This research aims to investigate second leg home advantage in football. UEFA Champions League quarter-finals and semi-finals from 1954-55 season to 2013-14 season were analyzed, adding up to 642 matches and 336 playoffs. A non-parametric binomial contrast was applied to determine the influence of playing at home, while the binary logistic regression was used to show the explanatory proportion of goals after winning the qualifying round following the first confrontation. The main results showed that, in quarter-finals, local teams (second match) passed the qualifying round at higher rates than in semi-finals, in which visiting teams (second match) were classified at higher rates. From a strategic point of view, a better matchup game model and the selection of an alignment more consistent with the order of two-legged ties, will be some of the utilities offered by this work for coaches.
\end{abstract}

Key words: home advantage; football; championship; UEFA 
Pic, M. y Castellano, J. (2016), Efecto de la localización del partido en eliminatorias de ida y vuelta de la UEFA Champions League. RICYDE. Revista internacional de ciencias del deporte. 44(12), 149-163.

\section{Introducción}

L a ventaja de jugar en casa o Home Advantage (HA, en el término anglosajón) ha sido objeto de estudio desde hace varias décadas (Pollard, 1986; Courneya y Carron, 1992) para conocer si existe asociación en el porcentaje de victorias conseguidas por el equipo local sobre los visitantes. La ventaja de jugar en casa (Nevill y Holder, 1999) en deportes de equipo puede situarse alrededor del 60\% (Jamieson, 2010), habiéndose particularizado estudios en criquet (Morley y Thomas, 2005), baloncesto (García, Saéz, Ibañez, Parejo, y Cañadas, 2009), beisbol (Levernier y Barrilla, 2007), voleibol (Marcelino, Anguera, Sampaio, y Mesquita, 2009; Marcelino, Mesquita, Palao, y Sampaio, 2009), Rugby (Morton, 2006; Gómez, Pollard, y Luis-Pascual, 2011) o balonmano (Pollard y Gómez, 2012), entre otros.

En el ámbito del fútbol, la ventaja de jugar en casa para equipos profesionales de fútbol inglés, en el Reino Unido, ha sido estimada en torno al 64\% (Pollard, 1986), o casi el 62\% en un trabajo posterior (Pollard, 2006), aunque en un trabajo más reciente (Pollard y Gómez, 2014b), sobre una muestra de 157 países de todo el mundo, encontraron que en Europa el efecto $H A$ tiene diferente peso según el país que se analice. En Bosnia-Herzegovina, por ejemplo, el porcentaje es superior al 75\% (Pollard y Gómez, 2014b). Esta ventaja parece desvanecerse cuando el equipo local cambia su ubicación a un nuevo estadio al ser encontrados valores inferiores de $H A$ (Pollard, 2002). Menores proporciones de $H A$ fueron encontradas, también, en competiciones de fútbol femenino frente a competiciones de fútbol masculino (Pollard y Gómez, 2014a). Otros estudios en el ámbito del fútbol también encontraron este efecto del $H A$ de los equipos de casa con valores en torno al $62 \%$ (Clarke y Norman, 1995) o hasta un 69\% (Corneya y Carron, 1992) de victorias conseguidas.

Las causas que podrían explicar el concepto del $H A$ son inconclusas y puede tener una explicación multifactorial (Carron, Loughead, y Bray, 2005; Courneya y Carron, 1992; Legaz, Moliner, y Munguía, 2013; Nevill y Holder, 1999; Nevill, Newell, y Gale, 1996; Pollard, 2008; Pollard y Pollard, 2005). Desde el modelo explicativo de $H A$, de Courneya y Carron (1992) se propusieron cinco factores, aunque posteriormente Pollard y Pollard (2005), aludieron a la interacción de hasta siete causas que podrían explicar el $H A$ : psicológicos, tácticos, territorialidad, familiaridad con el lugar, parcialidad del árbitro, apoyo del público y viajes previos al partido. Page y Page (2010) argumentaron que las relaciones entre los factores están desigualmente distribuidas e interaccionan entre sí. Así, factores como la parcialidad arbitral estaría condicionada por el apoyo del público. En este sentido, Nevill, Balmer, y Williams (2002) midieron la relación entre el acierto del árbitro y el ruido provocado por los seguidores de los equipos locales o densidad de público asistente, pudiendo provocar decisiones erróneas en los árbitros, señalando menos conductas antirreglamentarias al equipo local que al equipo visitante. En cualquier caso, son requeridas más evidencias científicas que lo corroboren (Agnew y Carron, 1994; Pollard, 1986). Por otro lado, otros factores como los psicológicos (Sánchez, González, Ruiz, San-Juan, Abando, De Nicolás, y García, 2001) podrían estar relacionados con la familiaridad (Barnett y Hilditch, 1993), viajes previos para llegar al lugar del partido (Clarke y Norman, 1995) y/o el apoyo del público (Nevill y col., 2002), afectando todos ellos al fenómeno de jugar en casa. Probablemente, los entrenadores transmitan o los jugadores asuman estrategias más ambiciosas al jugar como local, pudiendo ser el factor táctico-estratégico clave a nivel profesional (Staufenbiel, Lobinger, y Strauss, 2015). 
Pic, M. y Castellano, J. (2016), Efecto de la localización del partido en eliminatorias de ida y vuelta de la UEFA Champions League. RICYDE. Revista internacional de ciencias del deporte. 44(12), 149-163.

En este sentido, Schwartz y Barsky (1977) ya remarcaron la idea que los equipos locales suelen competir de manera más efectiva en relación a las acciones ofensivas que sus rivales visitantes, quizá condicionados por las expectativas de los entrenadores (Staufenbiel y col., 2015). Finalmente, otro factor asociado a la ventaja de jugar en casa es la territorialidad (Neave y Wolfson, 2003) entendida como la ventaja de jugar en una zona geográfica afín al grupo y su identidad cultural.

Recientemente el estudio del $H A$ ha sido investigado en las eliminatorias de ida y vuelta en el fútbol profesional denominada second leg home advantage (Eugster, Gertheiss, y Kaiser, 2010). En este estudio se analizaron los dieciseisavos, cuartos y semifinales desde la temporada 1994-95 hasta 2009-10 de partidos de la actual Champions League, distinguiendo a su vez dos períodos, tratando de desvelar si ser campeón de grupo facilitaría alcanzar un resultado positivo en el segundo partido, no existiendo diferencias estadísticamente significativas entre jugar el segundo partido como local o visitante en cuartos de final. En semifinales no hubo resultados que hicieran presumir ventaja alguna por jugar en casa. Un efecto de mayor tamaño pudo comprobarse en cambio, para dieciseisavos de final. Ningún resultado significativo se desprendió cuando la variable calidad del rival, medido a partir del coeficiente $U E F A$, fue incluida (Eugster y col., 2010).

Los investigadores Page y Page (2007), analizaron un total de 6.182 eliminatorias de ida y vuelta, durante el período que comprendió desde 1955 hasta 2006 en distintas eliminatorias europeas, confirman la existencia de ventaja significativa al jugar el segundo partido en casa, y concluyeron que en la actualidad existe un mayor equilibrio entre las eliminatorias exitosas afrontando el segundo partido fuera de casa. También, partiendo con unas premisas similares (Lidor y col., 2010) reforzaron la existencia de $H A$ en eliminatorias compuestas por dos partidos. En un estudio reciente (García, Gómez, Lago, y Ibañez, 2015) donde se analizaron 97 enfrentamientos pertenecientes a la fase de eliminatorias de la UEFA Champions League en el período comprendido entre los años 2009 y 2013 ha sido sugerida la importancia de la localización del partido (casa o fuera) así como marcar el primer gol del partido, para pasar las rondas de dicha competición. El marcador de baja puntuación como es el fútbol (Castellano, 2008) puede ser una de las razones que justifiquen la importancia de marcar primero. Además de la localización y el hecho de marcar primero, deben considerarse los efectos interactivos con otras variables como el nivel de los equipos o la diferencia de goles del partido de ida, que podrían aumentar o hacer decrecer las probabilidades de que un equipo pase la eliminatoria (García y col., 2015). Todavía se desconoce si estas variables tienen un comportamiento diferente dependiendo de la ronda de la eliminatoria (cuartos o semifinales) del campeonato.

Un aspecto de peso en un sistema de competición por eliminatoria (enfrentamientos de ida y vuelta o doble partido) otorga, en caso de empate, más valor a los goles marcados fuera de casa. Esta solución aplicada por la $U E F A$ en las eliminatorias de partidos de Champions League frente al empate, sigue vigente desde los años 60, dando así por ganador al equipo más goleador fuera de casa. Parece lógico pensar que el valor doble de los goles en campo contrario (en caso de empate) puede despertar interés por descubrir en qué medida se ve afectado el $H A$ cuando de este tipo de eliminatorias se trata, a sabiendas que mientras el objetivo del primer partido puede ser el de sacar una cierta renta, en el segundo partido se trata de pasar la eliminatoria (Page y Page, 2007) y, por tanto, pueda resultar exitoso también perder. 
Pic, M. y Castellano, J. (2016), Efecto de la localización del partido en eliminatorias de ida y vuelta de la UEFA Champions League. RICYDE. Revista internacional de ciencias del deporte. 44(12), 149-163.

Por todo lo comentado anteriormente, el presente trabajo pretende un doble objetivo: 1) por un lado, conocer el efecto de jugar en casa en el primer o segundo partido en una eliminatoria de dos partidos de competición europea de fútbol profesional; y por otro lado, 2) conocer si los goles de margen marcados en el primer partido constituyen una ventaja apreciable respecto a la probabilidad de pasar de ronda.

\section{Método}

\section{Muestra}

Para llevar a cabo el estudio se analizaron 336 eliminatorias (642 partidos) de la Copa de Europa o UEFA Champions League, entre los períodos que comprenden desde 1954-55 hasta 2013-14, que hicieron un total de 56 temporadas analizadas. Los partidos fueron agrupados en las cinco décadas que abarca el periodo estudiado, distinguiéndose además cuando los partidos se correspondían con los cuartos de final y las semifinales. Fueron excluidas las temporadas 1991-92, 1992-93 y 1993-94, dado que el formato de competición cambió y los equipos que pasaban una serie de eliminatorias previas, formaban dos grupos de cuatro equipos cada uno, siendo los campeones de ambos grupos los finalistas. Por tanto, seguir un formato de clasificación distinto al actual, en relación a cuartos y semifinales, supuso motivo de exclusión.

En relación a los goles anotados, fueron tenidos en cuenta los goles marcados por los equipos en tiempos de prolongación extra o prórrogas pero no los goles marcados tras finalizar estos, es decir, los conseguidos en las tandas de penaltis.

\section{Variables}

La variable localización (casa y fuera) es la variable independiente del estudio. Además, se ha considerado también la variable eliminatoria, distinguiéndose: cuartos de final y semifinal. El resultado obtenido por el equipo tras jugar los dos partidos de la eliminatoria se corresponde con la variable dependiente éxito (pasar de ronda). Del mismo modo, se ha incluido la diferencia de goles entre los equipos en el primer enfrentamiento, esto es, los goles marcados por el equipo menos goles recibidos, que será utilizada como un predictor para conocer la probabilidad de pasar la eliminatoria.

\section{Procedimiento}

Los datos fueron obtenidos de la página oficial de la Federación Europea de Fútbol (http://es.uefa.com/), donde se seleccionaron únicamente aquellos partidos que cumplieron los criterios de inclusión. Seguidamente, fue calculado el porcentaje de éxito (pase de ronda) para los equipos que jugaron el primer partido de la eliminatoria como visitante. Cada partido fue considerada una unidad estadística independiente, se calculó la proporción de equipos que al jugar el primer partido como visitante, superaron la eliminatoria distinguiendo entre cuartos de final y semifinales. El hecho de que los equipos puedan modificar las alineaciones, el planteamiento de juego o incluso el entrenador, lleva a pensar que agrupar los datos por equipos podría no ser la mejor opción. La participación irregular de los equipos a lo largo de la historia también dificultaría este procedimiento y por estos motivos se optó por organizar los datos en torno a la independencia de cada partido. Una vez construida la matriz se aplicó una regresión logística binaria, que ha sido utilizada anteriormente en diversos trabajos de investigación (Balmer, Nevill, y Lane, 2005; Everston, 2007; Gómez, Lorenzo, Ibañez, y Sampaio, 2013; Vinson, Padley, Croad, Jeffreys, Brady, y James, 2013). Finalmente, al considerar en el estudio la diferencia de goles obtenida por los equipos tras el primer partido jugando como local y como visitante, las unidades resultantes del margen de goles se consideraron positivas si el equipo ganaba y negativas si el equipo perdía. 


\section{Análisis de datos}

En relación a la ventaja de jugar en casa o fuera, se aplicó un contraste no paramétrico binomial, donde la hipótesis nula fue que la proporción es igual a 0,5. El contraste no paramétrico binomial posibilita el contraste de hipótesis, considerando la hipótesis nula si la proporción poblacional es igual o superior al $50 \%(\Pi=0,5)$, y considerando la hipótesis alternativa si la proporción poblacional es inferior al $50 \%(\Pi \neq 0,5)$. De acuerdo al nivel de significación $(\alpha=0,05)$, si $\mathrm{p}<0,05$ se aceptaba la hipótesis alternativa y si $\mathrm{p}>0,05$ no podremos rechazar la hipótesis nula. Para el análisis estadístico de los datos se utilizó el programa estadístico Statistical Package for Social Sciences versión 18.

El estudio sobre la influencia del margen de goles (diferencia entre el número de goles marcados) sobre el contrario en el partido de ida y pasar de ronda se ha planteado mediante regresión logística binaria. La variable dependiente es pasar la eliminatoria. La variable independiente o covariable, es el margen de goles, que podrá ser 0 si ambos equipos han empatado, positiva si el equipo ha ganado y negativa si ha perdido. El modelo resultante permitirá estimar la influencia que los goles de ventaja tendrán sobre la probabilidad de pasar de ronda. Se remarca que solo se estudia el margen de goles, exclusivamente en el partido de ida como local o visitante. Esta diferenciación informará sobre en qué situación (primer partido como local o como visitante) son más rentables los goles de ventaja para pasar de eliminatoria. En todos los casos, se empleó esta codificación para la variable dependiente (y): 1) No pasa: valor interno 0; y 2) Pasa: valor interno 1. Con todo, el modelo de regresión resultante revelará la probabilidad de pasar de ronda en función de la covariable $(\mathrm{x})$, que resultara ser el número de goles de ventaja. La ecuación del modelo será: $\mathrm{P}(\mathrm{y}=1)=$ $1 /\left(1+\exp \left(-\beta_{0}-\beta_{1} * x\right)\right)$.

Además, son definidos de esta manera la especificidad y sensibilidad de los modelos: 1) Sensibilidad: capacidad del modelo para predecir correctamente que un equipo pasa de ronda; y 2) Especificidad: capacidad del modelo para predecir correctamente que un equipo no pasa de ronda. La significación de la constante y coeficiente del modelo se basa en el estadístico de Wald, estableciéndose el punto de corte en 0,5. Antes de llevar a cabo el cálculo del modelo de regresión, se llevó a cabo la prueba U de Mann-Whitney para comprobar la relación entre la covariable y la variable dependiente. También, a través de la prueba de Hosmer y Lemeshow se comprobó la adecuación de los datos al modelo. Por último, se llevó a cabo un análisis exploratorio de la variable suma total de goles marcados. En este análisis se compararán los guarismos obtenidos por los equipos según su situación.

\section{Resultados}

\section{La ventaja del local}

De las 336 eliminatorias analizadas, se ha obtenido una proporción muestral unitaria de 0,49 , con una significatividad de $\mathrm{p}=0,623$. Jugar el segundo partido en casa o el primer partido fuera no obtuvo significación estadística para pasar las eliminatorias.

En la tabla 1 queda recogido el tamaño muestral $(\mathrm{N}=$ total de equipos que jugaron el primer partido de la ronda fuera de casa), la proporción muestral unitaria (n. ${ }^{\circ}$ de estos equipos que han pasado de ronda dividido entre N) y el p-valor o significación bilateral del test. 
Pic, M. y Castellano, J. (2016), Efecto de la localización del partido en eliminatorias de ida y vuelta de la UEFA Champions League. RICYDE. Revista internacional de ciencias del deporte. 44(12), 149-163.

Tabla 1: Tamaño muestral (N), proporción muestral (PM) y nivel de significación $(p)$.

\begin{tabular}{|c|c|c|c|c|}
\hline Ronda & Periodo & $\mathrm{N}$ & PM. & $p$ \\
\hline \multirow{8}{*}{ Cuartos } & $1955-59$ & 20 & 0,45 & 0,824 \\
\hline & $1960-69$ & 40 & 0,53 & 0,875 \\
\hline & $1970-79$ & 40 & 0,55 & 0,636 \\
\hline & $1980-89$ & 40 & 0,50 & 1,000 \\
\hline & 1990-99 & 28 & 0,43 & 0,572 \\
\hline & 2000-09 & 40 & 0,53 & 0,875 \\
\hline & $2010-14$ & 16 & 0,63 & 0,454 \\
\hline & Global cuartos & 224 & 0,51 & 0,738 \\
\hline \multirow{8}{*}{ Semifinales } & $1955-59$ & 10 & 0,30 & 0,344 \\
\hline & $1960-69$ & 20 & 0,25 & 0,041 \\
\hline & $1970-79$ & 20 & 0,60 & 0,503 \\
\hline & $1980-89$ & 20 & 0,45 & 0,824 \\
\hline & $1990-99$ & 14 & 0,43 & 0,791 \\
\hline & 2000-09 & 20 & 0,55 & 0,824 \\
\hline & $2010-14$ & 8 & 0,25 & 0,289 \\
\hline & Global semifinales & 112 & 0,43 & 0,156 \\
\hline Global & & 336 & 0,49 & 0,623 \\
\hline
\end{tabular}

La significación estadística para alguna de las décadas fue inexistente, sin tener en cuenta la variable eliminatoria. Por tanto, el hecho de jugar el primer partido como visitante no afectaría para pasar de ronda, en cualquiera de ellas, en la UEFA Champions League.

Se puede comprobar que la inclusión de la variable eliminatoria no reporta significatividad estadística al planteamiento que relaciona jugar el segundo partido en casa con pasar la eliminatoria. En este sentido, tanto en cuartos de final como en semifinales se rechaza la posible vinculación entre el efecto de jugar en casa el segundo partido y el éxito en la eliminatoria ( $p=0,738$ en cuartos y $p=0,156$ en semifinales).

Teniendo en cuenta la proporción muestral unitaria se observa que la tendencia mostrada por los cuartos de final, con valores de 0,51 es distinta de semifinales con 0,43. El significado de las proporciones encontradas verifican que en cuartos de final resulta más asequible pasar de ronda al afrontar el segundo partido en casa mientras que en semifinales resultaría más rentable para los intereses de los equipos jugar fuera de casa el segundo partido. A continuación, se muestra la evolución por décadas condicionada al tipo de ronda. Es la década 1960-69 en semifinales significativa con un valor de 0,041 . 
Pic, M. y Castellano, J. (2016), Efecto de la localización del partido en eliminatorias de ida y vuelta de la UEFA Champions League. RICYDE. Revista internacional de ciencias del deporte. 44(12), 149-163.

Como puede observarse en semifinales resultó ser inusual que la proporción fuera mayor de 0,5 , sin embargo en cuartos de final se dio más frecuentemente. Se podría apuntar que las eliminatorias ofrecen una evolución temporal similar, con la excepción de dos momentos: 1) por una parte, el contraste en la década 1960-69 con un valor de proporción muestral de 0,53 en cuartos de final por 0,25 en semifinales para la misma década; 2) el segundo punto de contraste es referido al período de 2010-14, siendo la proporción muestral en cuartos de final de 0,63 por 0,25 tomando las semifinales. Durante las cuatro décadas existentes entre estos dos mencionados períodos puede comprobarse una correlación entre las dos eliminatorias.

En relación al número de períodos o décadas que no alcanzan el límite de proporción muestral de 0,5 referidos a cuartos de final, encontramos un período (1955-59) y una década (1990-99) mientras las cinco agrupaciones temporales restantes, igualan o superan citado límite de proporción muestral. Sin embargo, las semifinales evidencian este mismo hecho en dos períodos $(1955-59,2010-14)$ y tres décadas $(1960-69,1980-89$ y 1990-99) por debajo de una proporción muestral de 0,5 .

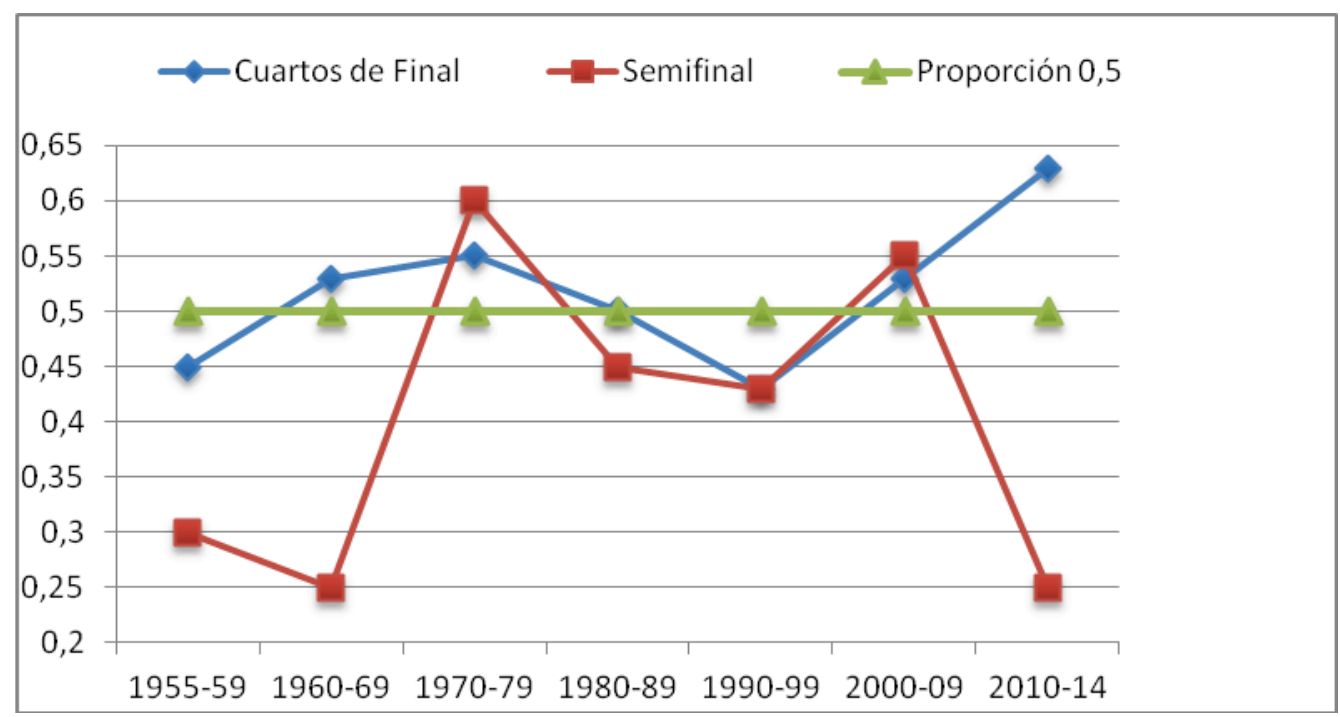

Figura 1. Evolución de la proporción muestral por décadas-períodos para cuartos de final y semifinal de la UEFA Champions league.

\section{La ventaja del gol}

En la siguiente tabla (tabla 2) es comprobada la significación entre la covariable y la variable dependiente en cuartos, como local $(z=-11,495, p<0,001)$ y como visitante $(z=-11,366, p$ $<0,001)$; en semifinales, como Local $(z=-9,169, p<0,001)$ y como visitante $(z=-9,211, p<$ $0,001)$.

La prueba de Hosmer y Lemeshow no presentó en ninguno de los casos significación estadística; en cuartos, como local $(\mathrm{p}=0,797)$ y como visitante $(\mathrm{p}=0,598)$; en semifinales, como local $(\mathrm{p}=0,172)$ y como visitante $(\mathrm{p}=0,330)$, con lo que es rechazada la hipótesis nula, advirtiendo que el modelo estaría bien ajustado. El coeficiente B para la covariable es significativo, y el intervalo de confianza para el $\operatorname{Exp}(B)$ no contiene el valor uno. Por tanto, la variable independiente tiene una influencia significativa en la ocurrencia del suceso. 
Pic, M. y Castellano, J. (2016), Efecto de la localización del partido en eliminatorias de ida y vuelta de la UEFA Champions League. RICYDE. Revista internacional de ciencias del deporte. 44(12), 149-163. http://dx.doi.org/10.5232/ricyde2016.04405

Tabla 2: Significación de la ventaja de jugar en casa (goles) según eliminatoria y localización del primer partido.

\begin{tabular}{|c|c|c|c|c|c|c|c|c|}
\hline \multirow{2}{*}{ Ronda } & \multirow{2}{*}{$\begin{array}{c}\text { Situación } \\
\text { equipo } \\
\text { en } \\
\text { primer } \\
\text { partido }\end{array}$} & \multirow{2}{*}{$\begin{array}{c}\text { Significación } \\
\text { para B }\end{array}$} & \multirow{2}{*}{$\operatorname{Exp}(B)$} & \multicolumn{2}{|c|}{$\begin{array}{c}\text { I.C. 95\% para } \\
\text { EXP(B) }\end{array}$} & \multirow{2}{*}{$\begin{array}{c}\text { R2 de } \\
\text { Nagelkerke }\end{array}$} & \multirow{2}{*}{ Sensibilidad } & \multirow{2}{*}{ Especificidad } \\
\hline & & & & Inf. & Sup. & & & \\
\hline \multirow{2}{*}{ Cuartos } & Local & $\mathrm{p}<0,001$ & 2,396 & 1,839 & 3,120 & 0,345 & 55,7 & 85,8 \\
\hline & Visitante & $\mathrm{p}<0,001$ & 2,200 & 1,715 & 2,822 & 0,308 & 82,0 & 62,7 \\
\hline \multirow{2}{*}{ Semifinal } & Local & $p<0,001$ & 2,530 & 1,700 & 3,764 & 0,332 & 82,0 & 62,7 \\
\hline & Visitante & $\mathrm{p}<0,001$ & 2,760 & 1,808 & 4,211 & 0,330 & 66,7 & 81,3 \\
\hline
\end{tabular}

A continuación se presentan los modelos que explican el valor de los goles del primer partido para pasar o no la eliminatoria.

Para los equipos que juegan el primer partido en casa (cuartos de final).

$P(y=1)=\frac{1}{1+\exp (1,091-, 874 * x)}$

Para los equipos que juegan el primer partido como visitante (cuartos de final):

$P(y=1)=\frac{1}{1+\exp (-, 725-, 788 * x)}$

Para los equipos que juegan el primer partido en casa (semifinal)

$P(y=1)=\frac{1}{1+\exp (, 651-, 928 * x)}$

Para los equipos que juegan el primer partido como visitante (semifinal):

$P(y=1)=\frac{1}{1+\exp (-, 539-1,015 * \mathrm{x})}$

La interpretación del $\operatorname{Exp}(\mathrm{B})$, odds ratio, o razón de ventajas, es la siguiente para cuartos de final:

- Por cada gol de ventaja que consiga el equipo local en el primer partido, la probabilidad de pasar de ronda aumenta 2,396 veces (un 240\%).

- Por cada gol de ventaja que consiga el equipo visitante en el primer partido, la probabilidad de pasar de ronda aumenta 2,200 veces (un 220\%).

La interpretación del $\operatorname{Exp}(\mathrm{B})$, odds ratio, o razón de ventajas, es la siguiente para las semifinales:

- Por cada gol de ventaja que consiga el equipo local en el primer partido, la probabilidad de pasar de ronda aumenta 2,530 veces (un 253\%).

- Por cada gol de ventaja que consiga el equipo visitante en el primer partido, la probabilidad de pasar de ronda aumenta 2,760 veces (un $276 \%$ ). 
Pic, M. y Castellano, J. (2016), Efecto de la localización del partido en eliminatorias de ida y vuelta de la UEFA Champions League. RICYDE. Revista internacional de ciencias del deporte. 44(12), 149-163.

\section{La ventaja de la diferencia de goles}

A continuación es mostrada una panorámica anacrónica de los goles marcados por los equipos en la UEFA Champions League durante los períodos comprendidos en la unidad muestral. El promedio de goles conseguidos por los equipos en cuartos de semifinal es de 5,4 goles por eliminatoria (1.220 goles en 224 eliminatorias) frente a 4,8 goles en semifinales (543 goles en 112 eliminatorias).

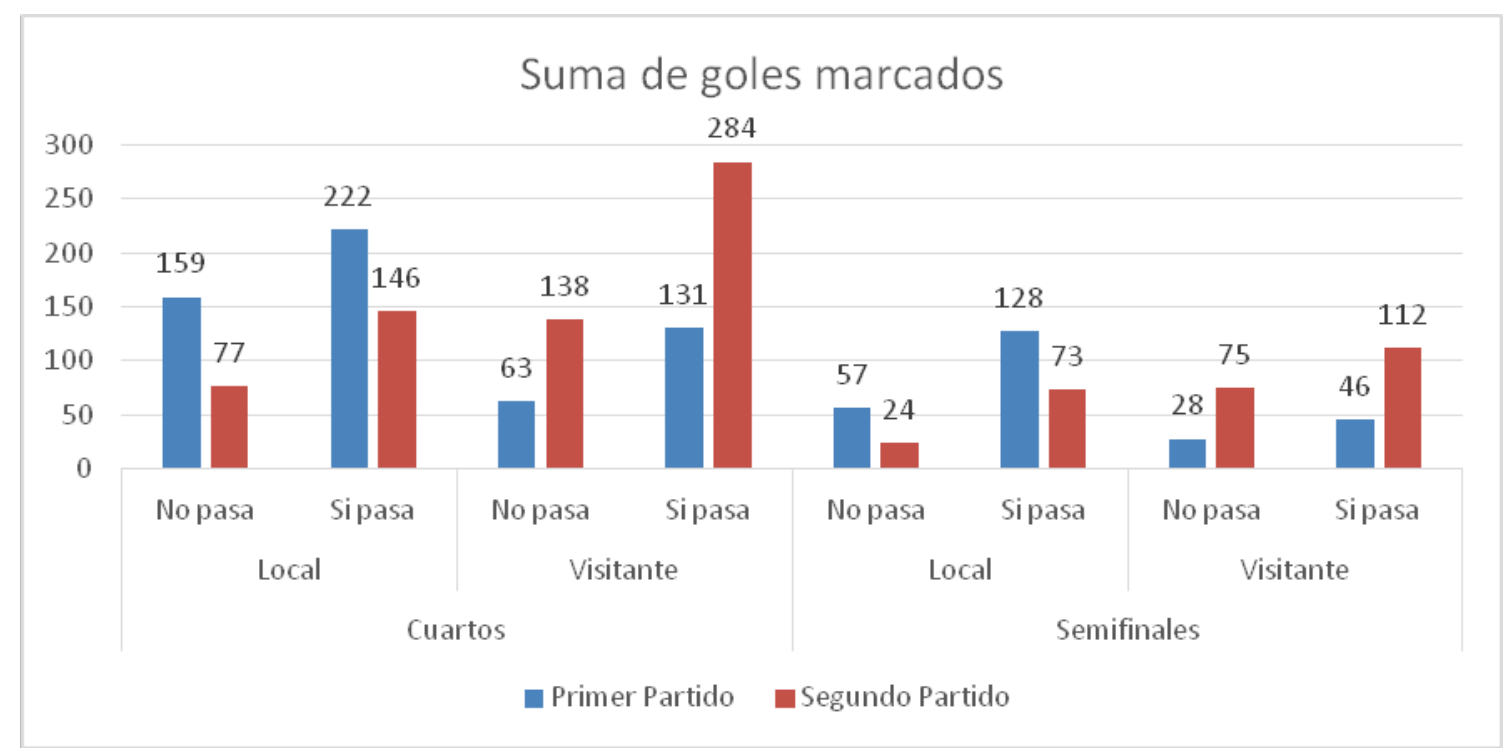

Figura 2. Goles marcados por los equipos según: eliminatoria (cuartos de final-semifinal), partido idapartido vuelta (primer partido-segundo partido), localización (local-visitante) y éxito (pasa o no pasa eliminatoria).

Nota: local es el equipo que compite en casa su primer partido y, visitante es el equipo que compite en casa su segundo partido.

Respecto a las semifinales, puede verse en la figura 2 que los equipos visitantes no son tan anotadores (261 goles) como los equipos locales (282 goles). Los equipos que pasan la eliminatoria como locales obtienen 201 goles, por 158 los visitantes. Puede ser comprobado que el rol visitante (segundo partido en casa) supone anotar menos goles después del primer o segundo partido para pasar la eliminatoria.

Con relación a los cuartos de final, los equipos locales (primer partido en casa) no son tan anotadores como los visitantes (segundo partido en casa) 604 goles frente a 616 goles, respectivamente. La diferencia entre empezar el primer partido como local o visitante en términos de goles supone pasar la eliminatoria como local con 385 goles pero 415 como visitante. Jugar la eliminatoria como local y pasar la eliminatoria supera en número de goles a los visitantes que no pasan la eliminatoria en su segundo partido (146 goles y 138 goles respectivamente). Este número de goles cambiará en semifinales.

Comparando ambas eliminatorias podría ser señalado que en semifinales se anotan menos goles que en cuartos de final (proporcionalmente). Comprobamos diferencias entre cuartos de final y semifinal en el número de goles obtenidos al afrontar el segundo como local, dado que serían constatados 284 goles obtenidos en cuartos de final por 112 goles procedentes de las semifinales. Otra diferencia remarcable advierte que ser local en cuartos de final durante el primer partido no supera en goles a los equipos visitantes en el segundo partido sin embargo en semifinales sería distinto es decir, los equipos locales que pasan la eliminatoria en el primer partido, obtienen más goles (128 goles) que los equipos visitantes en el segundo partido, es decir al jugar el primer partido fuera y el segundo como local (112 goles). 
Pic, M. y Castellano, J. (2016), Efecto de la localización del partido en eliminatorias de ida y vuelta de la UEFA Champions League. RICYDE. Revista internacional de ciencias del deporte. 44(12), 149-163.

\section{Discusión}

En este trabajo se han estudiado los efectos de jugar el primer o segundo partido de las eliminatorias de la UEFA Champions League como local o visitante. Se ha descrito el $H A$ mediante el análisis de equipos actuando como locales o visitantes. Además, fue estudiada la diferencia de goles marcados por los equipos en los partidos de ida de la eliminatoria para conocer la influencia sobre el éxito de seguir en competición.

Los resultados del presente estudio no confirman estadísticamente el argumento alineado con la supuesta ventaja de jugar el segundo partido en casa para pasar la eliminatoria, en la línea de lo mostrado en trabajos anteriores (García y col., 2015) donde variables como marcar primero o diferencia de goles del primer partido puede tener una influencia decisiva en el éxito de la eliminatoria. Sin embargo, una tendencia distinta para cuartos de final respecto a la semifinal podría poner de manifiesto la influencia de la localización en la resolución de la eliminatoria y constituir una fuente de explicación plausible para ayudar a seguir completando el círculo explicativo de las competiciones a doble partido y los efectos del $H A$. Esta tendencia será discutida de acuerdo con el éxito mostrado por los equipos para pasar o no la eliminatoria, así como la contribución de los resultados, para cuestionar paralelamente el porqué del valor doble de los goles anotados fuera de casa.

A partir del presente estudio, se apoyaría que no es ventajoso afrontar el segundo partido en casa para pasar de ronda. Cuando se diferenció cuartos de final de semifinales de la UEFA Champions League esta tendencia persistió. Una única década 1960-69 en semifinales, fue avalada por resultados estadísticamente significativos $(0,041)$ y una proporción muestral de 0,25 . Este resultado que señala como favorito al equipo que juega el segundo partido en casa podría ser debido a factores aleatorios. En cualquier caso, los resultados apuntan a que probablemente no sea ventajoso afrontar el segundo partido en casa. La existencia de marcadas tendencias en los períodos 1955-59 y 2010-14 y las décadas 1980-89 y 1990-99 sugiere considerar que al menos en semifinales de la UEFA Champions League más equipos que jugaron el segundo partido fuera de su estadio, continuaron en el campeonato. Sin embargo, otros estudios han mostrado conclusiones opuestas (Lidor y col., 2010; Page y Page, 2007). En el estudio de Page y Page (2007), tras analizar 6.182 eliminatorias correspondientes a tres competiciones de alto nivel de fútbol europeo, relacionaron significativamente el éxito de eliminatorias a doble partido con jugar el segundo partido en casa. Los investigadores no especificaron las eliminatorias estudiadas, pero podríamos estar ante una muestra compuesta en su mayoría de eliminatorias alejadas de las fases finales y, por tanto, un mayor número de enfrentamientos con los distintos niveles de los equipos. Con todo, el presente estudio coincidiría con el diagnóstico realizado por dichos autores en relación con la marcada desaceleración de $H A$, sobretodo tras los últimos 14 años de competición en la UEFA Champions League. En el estudio de Lidor y col. (2010) a pesar de confirmar la existencia de $H A$, es remarcado que tanto en semifinales como cuartos de final de la UEFA Champions League (1994-95 hasta 2006-2007) no existió sostén estadístico para esgrimir que jugar el segundo partido en casa supone una ventaja. García y col. (2015) coinciden con estos resultados.

En este sentido en relación a la figura 1, uniendo las eliminatorias para la década 2000-09 y período $2010-14$ se estimó una proporción muestral unitaria de $(0,53$ y 0,50$)$ respectivamente. De esta forma, se podría argumentar una mayor probabilidad de pasar de ronda para los equipos que juegan su segundo partido fuera de casa, aunque por un margen reducido. Sin embargo, si no se considera el tipo de eliminatoria, el balance de equipos clasificados para la siguiente fase en el período que comprende desde 2000-14 sería mayor, del lado de equipos que juegan su segundo partido fuera de casa. Eugster, Gertheiss, y Kaiser (2010) también 
Pic, M. y Castellano, J. (2016), Efecto de la localización del partido en eliminatorias de ida y vuelta de la UEFA Champions League. RICYDE. Revista internacional de ciencias del deporte. 44(12), 149-163.

coincidieron con las tendencias del presente estudio, considerando ventajoso jugar el segundo partido en casa para cuartos de final pero no así para las semifinales de la UEFA Champions League. A diferencia de estas fases, siguiendo a los mismos autores, para dieciseisavos de final de la misma competición, la ventaja de jugar en casa incrementó su efecto, lo cual podría atender al desequilibrio más evidente entre equipos que se enfrentan y que van equilibrándose a medida que avanzan las eliminatorias.

Quizá los momentos decisivos de la alta competición son vividos de manera distinta por los jugadores, y conforme van pasando eliminatorias, el apoyo del público local podría llegar a ser insuficiente en semifinales (García y col., 2015). Las eliminatorias suelen decidirse en el segundo partido, mientras que en el primero se busca obtener un resultado ventajoso (Page y Page, 2007), y, probablemente, sea esta una característica que condiciona el $H A$ en partidos de eliminatoria a diferencia de los campeonatos de liga regular.

Por su parte, el número de goles marcados por los equipos en el primer partido, se mostró significativo para los equipos locales y visitantes $(\mathrm{p}<0,001)$, también según la eliminatoria (Tabla 2). Esta ventaja en el gol average ya ha sido estudiada previamente (Barnett y Hilditch, 1993; Clarke, 2005; Clarke y Norman, 1995; Pollard y Gómez, 2009; Pollard, 1986; Pollard, 2002; Pollard, 2006; Pollard y Pollard, 2005). Sin embargo, estos hallazgos parecen ser insuficientes para explicar la complejidad del fenómeno de las eliminatorias de ida y vuelta, y el impacto que sobre ellas pudieran ejercer los goles marcados o encajados en casa o fuera en el primero o segundo partido. La inclusión de variables como la calidad de los equipos, el ser primero en marcar (García y col., 2015) o la asistencia de público, entre otros, podrían incrementar la capacidad predictiva del modelo de $H A$, basada en la interacción de factores (Pollard y Pollard, 2005). Estas variables no fueron incluidas en el presente estudio, lo cual es una limitación del mismo.

Uno de los indicadores que puede condicionar el $H A$ en eliminatorias a doble partido es el número de goles realizados por los equipos (también la diferencia de goles), es decir, la ventaja disponible al finalizar el primer partido, el de ida. El resultado del primer partido podría condicionar el planteamiento estratégico que los equipos tomarían para afrontar el segundo de los encuentros, donde, como ya se ha comentado anteriormente, el objetivo no es ganar sino pasar la eliminatoria (Page y Page, 2007). Parece que en cuartos de final los equipos locales en el primer partido obtendrían mayor ventaja por cada gol de ventaja que los equipos visitantes. Es decir, los primeros, rentabilizarían estos goles para tener mayores opciones de pasar la ronda. Sin embargo, en idéntica situación, pero en semifinales, serían los equipos visitantes en el primer enfrentamiento más beneficiados de los goles realizados fuera de casa, con el fin de tener mayores opciones de pasar la ronda. Esto significa que la situación en semifinales invirtió significativamente el resultado, advirtiéndose consecuentemente al igual que sucedía con el $H A$, efectos diferentes en función de la eliminatoria.

Quizás para discernir el alcance de los goles (Roffé, Llinás, Marcos, y García, 2007) sea necesario vislumbrar lo sucedido tras ambos encuentros. La figura 2 muestra como el $\mathrm{n} .^{\circ}$ de goles desciende en semifinales $(30,8 \%, 543$ goles) con respecto a los marcados en cuartos de final $(69,2 \%, 1.220$ goles), probablemente debido a una mayor igualdad entre los equipos. Esto incrementa la importancia de cada gol en semifinales, en consecuencia, los goles anotados durante el segundo partido de las semifinales parece ser más crucial si cabe. Tras considerar que el número estimado de goles debería ser de 33,3\% del total en semifinales por el 66,6\% de cuartos de final se desprende que los goles marcados en semifinales se sitúan tres puntos porcentuales por debajo de lo esperado y en cuartos de final unos tres puntos por encima. Cabe destacar que mientras en semifinales jugar en casa el segundo partido y pasar la 
Pic, M. y Castellano, J. (2016), Efecto de la localización del partido en eliminatorias de ida y vuelta de la UEFA Champions League. RICYDE. Revista internacional de ciencias del deporte. 44(12), 149-163.

eliminatoria supuso un 70,8\% (112 goles) del total de goles visitantes, en cuartos de final supusieron el 68,4\% (284 goles) del número total de goles de los equipos que jugaron en casa el segundo partido. Así, para los equipos que jugaron en casa el segundo partido de semifinales, los goles fueron más determinantes que para los cuartos de final. Quizás, por tanto, no solo el tipo de competición, liga o copa, condicionaría el peso de variables como calidad del oponente, lugar o marcar primero en las probabilidades de éxito, también y, dentro de una misma competición, la proximidad con el final del campeonato podrían tener influencia.

Entendemos que la descripción diacrónica del número de goles anotados bajo las distintas variables del estudio pone de relieve que no estamos ante sucesos aleatorios. Ya fue estudiada la evolución del fútbol (Castellano, Perea, y Hernández, 2008) y pudo comprobarse una disminución del número de goles por partido en los mundiales desde 1930 hasta 2006. Si bien, el mayor componente azaroso del estudio pareció surgir de jugar el segundo partido en casa o lejos del estadio, también en cualquier caso, debería ser puesto en valor para la construcción de un modelo de interacción de factores (Pollard y Pollard, 2005) que proporcione explicación al fenómeno. Por tanto, para el adecuado cálculo de la 'ventaja en casa' (Pollard y Gómez, 2012) sería primordial desvelar los factores que podrían condicionar dicho fenómeno. Como ejemplo de variables trascendentes podría darse cita el apoyo del público (Nevill y col., 2002), pero también el género (Pollard y Gómez, 2014a), el país del campeonato (Pollard y Gómez, 2014b), u otras, que no han sido identificadas, por el momento, o combinadas con las anteriores variables.

Este singular modelo de competición, demanda cierta especificidad, ya que disponer del resultado del primer partido podría llegar a condicionar las variables externas, como el apoyo del público, o las internas de los equipos, donde las directrices marcadas por los entrenadores pueden ser interpretadas por los jugadores ante un resultado equilibrado, desequilibrado o muy holgado. En qué medida un resultado puede convertirse en condición, necesita ser esclarecido y cómo puede afectar a la estrategia (Pollard, 2008) de los encuentros de vuelta.

La inclusión de covariables 'cambios reglamentarios' mediante análisis de series temporales se ofrece como un reto de interés para futuros trabajos y al mismo tiempo, una posible limitación del trabajo que presentamos. En lo concerniente al respaldo estadístico, sí hemos podido evidenciar la tendencia de la proporción muestral a un comportamiento distinto al tratarse de cuartos de final o semifinales. También podría ser oportuno, avivar la discusión en torno al valor doble de los goles marcados en el terreno del rival. Convendría señalar la necesidad de explorar amplias muestras incluyendo la variable ronda, debido a que posiblemente, la presión extra que supone jugar el segundo partido de una eliminatoria de semifinales de la Champions League en casa, parece disponer de menores posibilidades de clasificación. Este hecho parece insólito y contrasta con los resultados obtenidos en eliminatorias de cuartos de final de la UEFA Champions League, en la que la ventaja de jugar en casa el segundo partido, sí afectó positivamente para pasar de ronda.

\section{Conclusiones}

La principal conclusión que subyace a la investigación planteada, apuntaría al comportamiento irregular del $H A$ al tratar la variable eliminatoria, resultando beneficioso afrontar los cuartos de final como equipo local en el segundo partido. Por otro lado, también las leves tendencias apreciadas en semifinales sugieren que puede resultar ventajoso jugar el segundo partido como equipo visitante. De entre las aplicaciones prácticas de los resultados del estudio podrían destacarse: ayudar a los entrenadores con el diseño de alineaciones o 
Pic, M. y Castellano, J. (2016), Efecto de la localización del partido en eliminatorias de ida y vuelta de la UEFA Champions League. RICYDE. Revista internacional de ciencias del deporte. 44(12), 149-163.

elegir modelos de juego o estrategias más o menos conservadoras acorde al orden de los enfrentamientos en un modelo de competición de ida y vuelta.

\section{Referencias}

Agnew, G. A., \& Carron, A. V. (1994). Crowd effects and the home advantage. International Journal of Sport Psychology, 25, 53-62.

Balmer, N. J.; Nevill, A. M., \& Lane, A. M. (2005). Do judges enhance home advantage in European championship boxing?. Journal of Sports Sciences, 23(4), 409-416. http://dx.doi.org/10.1080/02640410400021583

Barnett, V., \& Hilditch, S. (1993). The effect of an artificial pitch surface on home team performance in football (soccer). Journal of the Royal Statistical Society, 156(1), 39 -50. http://dx.doi.org/10.2307/2982859

Carron, A. V., Loughhead, T. M., \& Bray, S. R. (2005). The home advantage in sport competitions: Courneya and Carron's (1992) conceptual framework a decade later. Journal of Sports Sciences, 23(4), 395- 407.

http://dx.doi.org/10.1080/02640410400021542

Castellano, J. (2009). Conocer el pasado del fútbol para cambiar su futuro. Acción Motriz, 2, 1-19.

Castellano, J.; Perea, A., y Hernández-Mendo, A. (2008). Análisis de la evolución del fútbol a lo largo de los mundiales. Psicothema, 20(4), 928-932.

Clarke, S. R. (2005). Home advantage in the Australian football league. Journal of Sports Sciences, 23(4), 375-385.

http://dx.doi.org/10.1080/02640410500074391

Clarke, S. R., \& Norman, J. M. (1995). Home advantage of individual clubs in English soccer. The Statistician, 44(4), 509-521. http://dx.doi.org/10.2307/2348899

Courneya, K. S., \& Carron, A. V. (1992). The home advantage in sport competitions: A literature review. Journal of Sport and Exercise Psychology, 14, 13-27.

Eugster, M. J. \& Gertheiss, J., \& Kaiser, S. (2010). Having the second leg at home advantage in the UEFA champions league knockout phase? Journal of Quantitative Analysis in Sports, 7(1), 1-9.

Everson, P. (2007). Teaching regression using American football scores. International Statistical Institute, 56th Session. Swarthmore (USA).

García, J.; Sáez, J.; Ibañez, S. J.; Parejo, I., \& Cañadas, M. (2009). Home Advantage in ACB league 2007-2008. Revista Psicología del Deporte, 18 (suppl), 331-335.

García, J.; Gómez, M.; Lago, C., \& Ibáñez, J. (2015). Effect of match venue, scoring first and quality of opposition on match outcome in the UEFA Champions League. International Journal of Performance Analysis in Sport, 15(2), 527-539.

Gómez, M. A.; Lorenzo, A.; Ibañez, S. J., \& Sampaio, J. (2013). Ball possession effectiveness in men's and women's elite basketball according to situational variables in different game periods. Journal of sports sciences, 31(14), 1578-1587. http://dx.doi.org/10.1080/02640414.2013.792942

Gómez, M. A.; Pollard, R., \& Luis-Pascual, J. C. (2011). Comparison of the home advantage in nine different Professional team sports in Spain. Perceptual and Motor Skills, 113, 150-156.

http://dx.doi.org/10.2466/05.PMS.113.4.150-156 
Pic, M. y Castellano, J. (2016), Efecto de la localización del partido en eliminatorias de ida y vuelta de la UEFA Champions League. RICYDE. Revista internacional de ciencias del deporte. 44(12), 149-163.

Jamieson, J. P. (2010). Home field advantage in athletics: a meta-analysis. Journal of Applied Social Psychology, 40, 1819-1848.

http://dx.doi.org/10.1111/j.1559-1816.2010.00641.x

Lidor, R.; Bar-Eli, M.; Arnon, M., \& Bar-Eli, A. A. (2010). On the advantage of playing the second game at home in the knock out stages of European soccer cup competitions. International Journal of Sport and Exercise Psychology, 8(3), 312-325. http://dx.doi.org/10.1080/1612197X.2010.9671956

Legaz, A.; Moliner, D., \& Munguía, D. (2013). Home Advantage and Sports Performance: Evidence, Causes and Psychological Implications. Universitas Psychologica, 12(3), 933-943.

Levernier, W., \& Barilla, A. (2007). The home-field advantage in Major League Baseball. The Berkeley Electronic Press, 1-22.

Marcelino, R.; Mesquita, I.; Palao, J. M. \& Sampaio, J. (2009). Home Advantage in highlevel volleyball varies according to set number. Journal of Sport Science and Medicine, 8(3), 352-356.

Marcelino, R.; Anguera, M. T.; Sampaio, J., y Mesquita, I. (2009). Ventaja de jugar en casa en voleibol de alto rendimiento. Revista de psicología del deporte, 18(2), 181196.

Morley, B., \& Thomas, D. (2005). An investigation of home advantage and other factors affecting outcomes in English one-day cricket matches. Journal of sports sciences, 23(3), 261-268.

http://dx.doi.org/10.1080/02640410410001730133

Morton, R. H. (2006). Home advantage in southern hemisphere rugby union: national and international. Journal Sports of Science, 24(5), 495-499.

http://dx.doi.org/10.1080/02640410500189074

Neave, N., \& Wolfson, S. (2003). Testosterone, territoriality, and the 'home advantage'. Physiology and Behavior, 78(2), 269-275.

http://dx.doi.org/10.1016/S0031-9384(02)00969-1

Nevill, A. M.; Newell, S. M., \& Gale, S. (1996). Factors associated with home advantage in English and Scottish football matches. Journal of Sports Sciences, 14(2), 181-186. http://dx.doi.org/10.1080/02640419608727700

Nevill, A. M., \& Holder, R. L. (1999). Home advantage in sport: An overview of studies on the advantage of playing at home. Sports Medicine, 28(4), 221-236. http://dx.doi.org/10.2165/00007256-199928040-00001

Nevill, A. M.; Balmer, N. J., \& Williams A. M. (2002). The influence of crowd noise and experience upon refereeing decisions in football. Psychology of Sport and Exercise, $3(4), 261-272$.

http://dx.doi.org/10.1016/S1469-0292(01)00033-4

Page, K., \& Page, L. (2010). Alone against the crowd: Individual differences in referees' ability to cope under pressure".Journal of Economic Psychology, 31(2), 192-199. http://dx.doi.org/10.1016/j.joep.2009.08.007

Page, L., \& Page, K. (2007). The second leg home advantage: Evidence from European football cup competitions. Journal of Sports Sciences, 25(14), 1547-1556. http://dx.doi.org/10.1080/02640410701275219

Pollard, R. (1986). Home advantage in soccer: A retrospective analysis. Journal of Sports Sciences, 4(3) 237-248.

http://dx.doi.org/10.1080/02640418608732122 
Pic, M. y Castellano, J. (2016), Efecto de la localización del partido en eliminatorias de ida y vuelta de la UEFA Champions League. RICYDE. Revista internacional de ciencias del deporte. 44(12), 149-163.

Pollard, R. (2002). Evidence of a reduced home advantage when a team moves to a new stadium. Journal of Sports Sciences, 20(12), 969-973.

http://dx.doi.org/10.1080/026404102321011724

Pollard, R., \& Pollard, G. (2005). Home advantage in soccer: A review of its existence and causes. International Journal of Soccer and Science, 3(1), 28-38.

Pollard, R. (2006). Worldwide regional variations in home advantage in association football. Journal of Sports Sciences, 24(3), 231-240. http://dx.doi.org/10.1080/02640410500141836

Pollard, R. (2008). Home Advantage in Football: A current review of an unsolved puzzle. The Open Sports Sciences Journal, 1(1), 12-14. http://dx.doi.org/10.2174/1875399X00801010012

Pollard, R., \& Gómez, M. A. (2009). Home advantage in football in South-West Europe: Long-term trends, regional variation, and team differences. European Journal of Sport Science, 9(6), 341-352. http://dx.doi.org/10.1080/17461390903009133

Pollard, R., \& Gómez, M. A. (2012). Reassessment of home advantage in Spanish handball: comment on Gutiérrez et al (2012). Perceptual and Motor Skills, 115(3), 937-943. http://dx.doi.org/10.2466/06.05.PMS.115.6.937-943

Pollard, R., \& Gómez, M. A., (2014a). Comparison of home advantage in men's and women's football leagues in Europe. European Journal of Sport Science, 14, S77S84.

http://dx.doi.org/10.1080/17461391.2011.651490

Pollard, R., \& Gómez, M. A. (2014b). Components of home advantage in 157 national soccer leagues worldwide. International Journal of Sport and Exercise Psychology, 12(3), 218-233.

http://dx.doi.org/10.1080/1612197X.2014.888245

Pollard, R., \& Pollard, G. (2005). Home advantage in soccer: a review of its existence and causes. International Journal of Soccer and Science Journal, 3(1), 28-38.

Roffé, M.; Llinás, J.; Marcos, V., y García, A. (2007). Las crisis durante el juego: el gol psicológico en el fútbol. Revista de psicología del deporte, 16(2), 227-240.

Sánchez, A.; González, E.; Ruiz, M.; San-Juan, M.; Abando, J.; De Nicolás, L., y García, F. (2001). Estados de ánimo y rendimiento deportivo en fútbol: ¿existe la ventaja de jugar en casa? Revista de Psicología del Deporte, 10(2), 197-209.

Schwartz, B., \& Barsky, S. F. (1977). The home advantage. Social Forces, 55(3), 641661.

http://dx.doi.org/10.1093/sf/55.3.641

Staufenbiel, K.; Lobinger, B., \& Strauss, B. (2015). Home advantage in soccer-A matter of expectations, goal setting and tactical decisions of coaches?. Journal of sports sciences, 33(18), 1932-1941.

http://dx.doi.org/10.1080/02640414.2015.1018929

Vinson, D.; Padley, S.; Croad, A.; Jeffreys, M.; Brady, A., \& James, D. (2013). Penalty corner routines in elite women's indoor field hockey: Prediction of outcomes based on tactical decisions. Journal of sports sciences, 31(8), 887-893. http://dx.doi.org/10.1080/02640414.2012.757341 